\title{
Wnt signaling and stem cell control
}

\author{
Roel Nusse ${ }^{1}$ \\ ${ }^{1}$ Howard Hughes Medical Institute, Department of Developmental Biology, Stanford University School of Medicine, Stanford, CA \\ 94305, USA
}

Wnt signaling has been implicated in the control over various types of stem cells and may act as a niche factor to maintain stem cells in a self-renewing state. As currently understood, Wnt proteins bind to receptors of the Frizzled and LRP families on the cell surface. Through several cytoplasmic relay components, the signal is transduced to $B$-catenin, which then enters the nucleus and forms a complex with TCF to activate transcription of Wnt target genes. Wnts can also signal through tyrosine kinase receptors, in particular the ROR and RYK receptors, leading to alternative modes of Wnt signaling. During the growth of tissues, these ligands and receptors are dynamically expressed, often transcriptionally controlled by Wnt signals themselves, to ensure the right balance between proliferation and differentiation. Isolated Wnt proteins are active on a variety of stem cells, including neural, mammary and embryonic stem cells. In general, Wnt proteins act to maintain the undifferentiated state of stem cells, while other growth factors instruct the cells to proliferate. These other factors include FGF and EGF, signaling through tyrosine kinase pathways.

Keywords: Wnt, stem cells, self-renewal, signal transduction, niche

Cell Research (2008) 18:523-527. doi: 10.1038/cr.2008.47; published online 8 April 2008

How can the differentiation and self-renewal of stem cells, including embryonic stem cells, be controlled? This is a central question in applying stem cell technology to regenerative medicine. From embryological experiments, it has become clear that commitment to developmental lineages is a stepwise process, involving a succession of changes in cell state. In order to guide stem cells towards defined fates, it will be essential to know how these changes are regulated and how to manipulate the cells in such a way that they change in a predictable and reproducible way. Factors that define or control developmental stages have been discovered, and include transcriptional regulators. Ideally however, the state of differentiation of stem cells is accomplished by supplying outside signals, extra-cellular factors, rather than genetic manipulation. In vivo, these signals and the micro-environment constitute a niche in which stem cells are present and compete for limiting concentrations of growth factors, thereby maintaining a balance between self-renewal and differentiation of the cells (Figure 1). The best candidate factors are the ones that regulate cell fate decisions in normal embryos and those include members of the BMP, Hedgehog, FGF and Wnt molecules, plus

Correspondence: Roel Nusse

Tel: +1-650-723-7769; Fax: +1-650-723-1399

E-mail: rnusse@stanford.edu small molecules such as retinoic acid. In that group, the class of Wnt proteins stands out because of numerous functions during development. Indeed, Wnt signaling and Wnt proteins are important for the maintenance of stem cells of various lineages. The classic example is in the digestive tract, where in the crypt of the colon the loss of transcription factor TCF4 leads to depletion of stem cells [1]. The Wnt pathway has also been implicated as a self-renewal signal in the hematopoietic system $[2,3]$. Alternatively, loss of the tumor suppressor APC or gain of $\beta$-catenin activity leads to excess of stem cells and cancer [4, 5]. In this review, I will give an overview of Wnt signaling, emphasizing the potential role for Wnt proteins as stem cell factors.

\section{Wnt signaling, Wnt proteins}

The Wnts comprise a large family of protein ligands that affect diverse processes such as embryonic induction, generation of cell polarity, and the specification of cell fate [6]. Wnts are defined by amino acid sequence rather than by functional properties [7, 8]. As many as 19 mammalian Wnt homologues are known and are expressed in temporalspatial patterns (www.stanford.edu/ rnusse/wntwindow. $\mathrm{html}$ ). Shared features of all Wnts include a signal sequence for secretion, several highly charged amino acid residues, and many glycosylation sites. Wnt proteins also display a characteristic distribution of 22 cysteine residues. Upon 


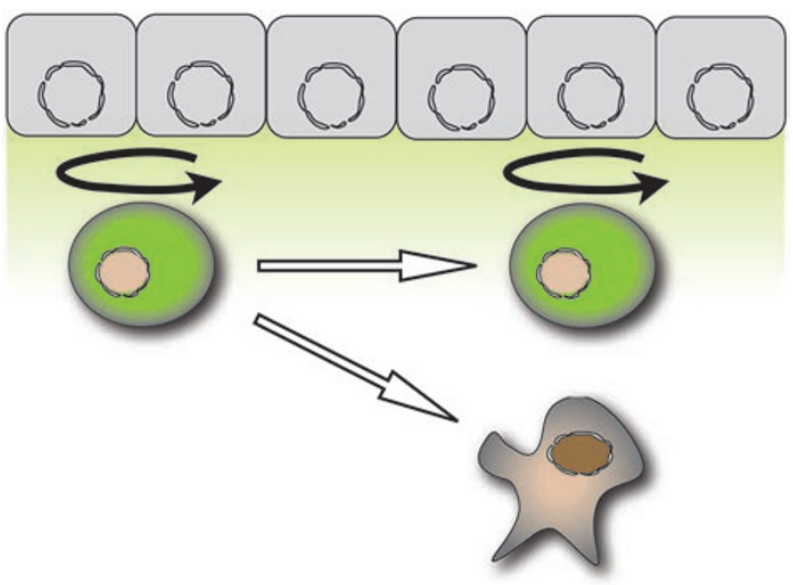

Figure 1 Model of a niche (upper layer of grey cells) maintaining nearby stem cells (green) in a self-renewing state. Cells outside of the direct contact with the niche will differentiate.

overexpression in tissue culture cells, several different $\mathrm{N}$ linked glycosylated intermediate Wnt protein products are observed in cell lysates [9-11], suggesting that Wnt protein processing and secretion are highly regulated processes.
The primary amino acid sequence of Wnts suggests that they should be soluble, but secreted Wnt proteins are hydrophobic and are mostly found associated with cell membranes and the extracellular matrix (ECM). Following the purification of active Wnt proteins, it was discovered why they are hydrophobic [3]: mass spectroscopy revealed that Wnt proteins are lipid modified by the attachment of a palmitate on the first conserved cysteine residue within the protein family and on a serine in the middle of the protein [12].

While palmitoylation has been shown to be necessary for Wnt signaling, the exact role of lipid modification remains unclear. A possible function is the targeting of Wnts to particular domains of the membrane. In addition, palmitoylation of Wnt proteins may be necessary for their glycosylation. This proposed role for palmitoylation during N-linked glycosylation might also aid in Wnt transport between cells as glycosylation might increase Wnt interactions with heparin sulfate proteoglycans (HSPGs) present on the surface of Wnt responding cells. Alternatively, the palmitate moiety could potentially anchor Wnt proteins into the membrane for sustained signaling. In stem cell biology, the interactions between Wnts and membrane are likely
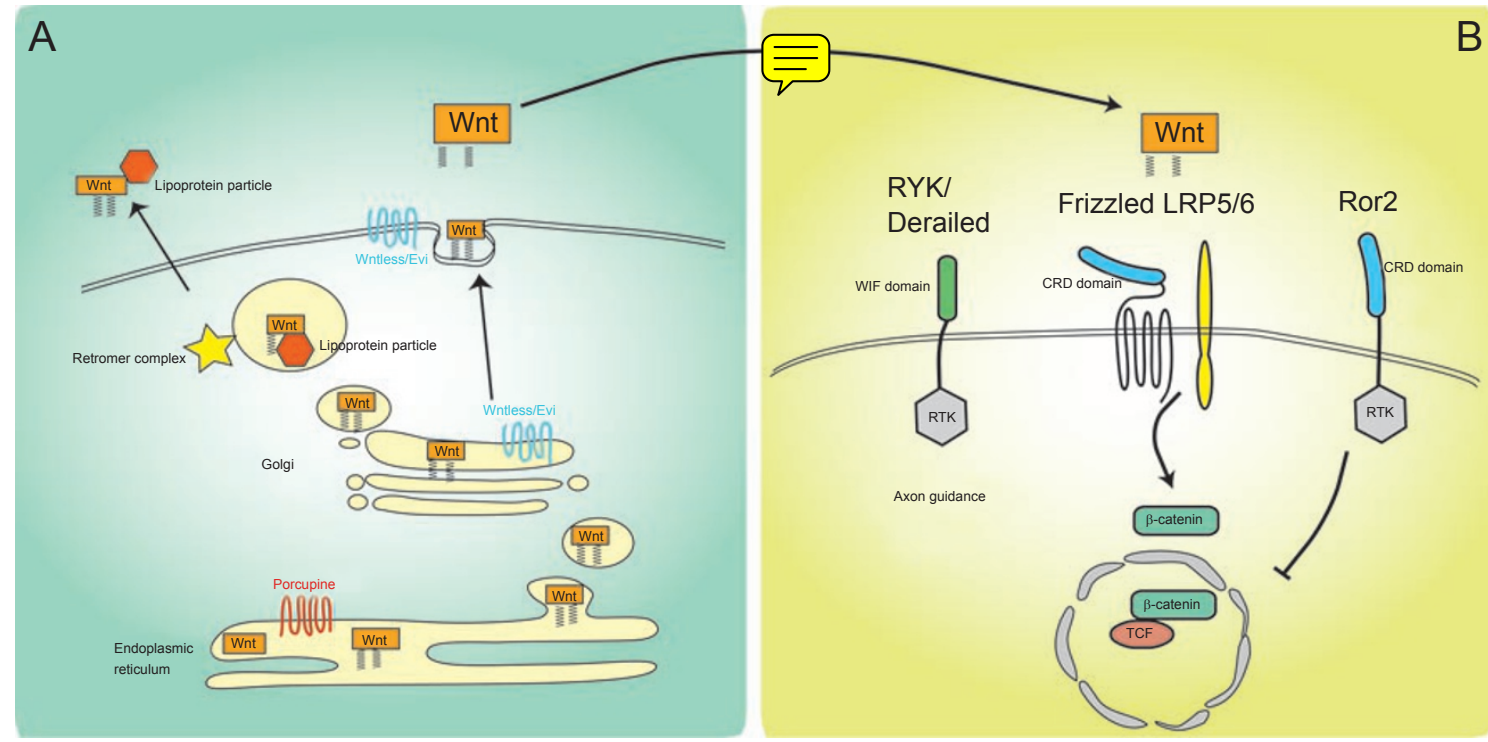

Figure 2 (A) In Wnt-producing cells, the Wnt protein becomes palmitoylated at 2 sites in the endoplasmic reticulum by the porcupine acyl-transferase. Further transport and secretion of the Wnt protein in secretory vesicles is controlled by the multi-pass transmembrane protein Wntless/Evi, which is present in the Golgi and/or on the plasma membrane. The retromer complex, including VPS35, may act within Wnt-producing cells to generate Wnt forms that can be transported outside cells, possibly in the form of a lipoprotein particle. (B) Wnts can interact with multiple receptors. These include the RYK/Derailed type receptors that have a tyrosine kinase motif and a WIF (Wnt inhibitory factor) ligand-binding domain. This receptor can mediate signaling during axon guidance in Drosophila. During Wnt/ $\beta$-catenin signaling, the Wnts interact with Frizzleds through the cysteine-rich domain (CRD). The LRP5/6 class of transmembrane proteins act as co-receptors, while in Xenopus axis formation FRL1/Cripto can acts as an additional co-receptor. Finally, Wnts can also interact with the Ror tyrosine kinases to mediate a signal that can inhibit $\beta$-catenin TCF activity in the nucleus. 
of great importance in understanding how niches control stem cell fate, commonly thought to be in close cell to cell configurations (Figure 1).

\section{Wnt secretion}

There are other proteins that might be involved in Wnt protein processing and secretion from producing cells (Figure 2). Genetic screens have identified the multipass transmembrane protein Wntless (Wls)/Evenness interrupted (Evi) to be required in the secretory pathway to promote the release of Wnts from producing cells [13]. In Drosophila and mammalian tissue culture, RNAi knock-down of $w l s /$ evi/mom-3 in cells overexpressing Wnt inhibits a Wnt-responsive luciferase reporter in co-cultured responding cells, showing that Wls/Evi acts in a cell non-autonomous fashion [14]. Lack of wls/evi inhibits Wnt transport to the surface of cells [15]. These phenotypes are similar to those observed in porcupine mutants. In contrast to Porcupine's function, however, the secretory activity mediated by Wls/Evi does not depend on the palmitoylation status of Wnt proteins: the secretion of Wnt proteins in which the palmitoylation site is mutated is still impaired in wls/evi mutants.

\section{Wnt extracellular transport}

Recently, large molecules required for lipid transport called lipoprotein particles have been implicated in moving Wnts and other lipid-modified proteins such as Hedgehogs (Figure 2). While previously postulated to be exosome-like particles, these structures termed "argosomes" are now thought to be exogenously derived lipoproteins [16].

A model is proposed wherein palmitoylated proteins associate with lipoprotein particles on the extracellular face of cells. Traffic of Wnt proteins from one cell to the next requires this association, as RNAi knock-down of Lipophorin, a Drosophila lipoprotein, narrows the range of $\mathrm{Wg}$ signaling in the wing disc [16]. It will be interesting to see what mechanism underlies these lipoprotein particles' aiding in Wnt transport and whether this mechanism of Wnt transport is conserved across all species.

In addition, transcytosis may regulate Wnt movement. In yeast, proteins of the retromer complex direct endosometo-Golgi retrieval of proteins. In vertebrate systems, the retromer complex functions in basal-to-apical transcytosis [17]. In Caenorhabditis elegans, the ortholog of the yeast retromer complex subunit $\mathrm{Vps} 35 \mathrm{p}$ is required for the longrange signaling capabilities of Egl-20 (C. elegans Wnt ortholog) $[18,19]$. It has been proposed that the retromer complex promotes the association of secreted Wnts with other proteins required for ligand transport, such as lipoprotein particles (Figure 2).

\section{Wnt reception}

The seven-pass transmembrane protein Frizzled (Fz) protein was the first receptor found to transduce a Wnt signal [20] (Figure 2). Fz proteins contain a large extracellular domain containing a conserved motif comprised of 10 cysteine residues called the cysteine-rich domain (CRD). The CRD domains from various $\mathrm{Fz}$ receptors have been shown to bind multiple Wnts with high affinity [21, 22]. At the cytoplasmic side, Fzs may interact directly with the Dishevelled protein, a known mediator of Wnt signaling [23].

Much remains to be discovered regarding the mechanism of Wnt receptor signaling in receiving cells (Figure 2). There is a requirement for the single-pass transmembrane proteins of the low-density lipoprotein (LDL) family called Lrp5 and -6 [24]. Following Wnt binding, it is thought that Fzs form a co-receptor complex with Lrp proteins to transduce the canonical Wnt signal [25]. Lrp5 and -6 proteins have a relatively small intracellular domain and a large extracellular domain containing several potential protein interaction domains [26]. Truncated proteins that lack the extracellular portion of the protein, but still contain the transmembrane and intracellular domains, produce a constitutively active canonical Wnt signal [27].

Interestingly, the Axin protein, a negative regulator of Wnt signaling, can bind to the cytoplasmic tail of LRP6, providing a mechanism by which Axin is released from $\beta$ catenin [28]. This interaction changes the fate of $\beta$-catenin; instead of being destroyed, it accumulates to execute Wnt-induced gene expression. The binding of Axin to the LRP6 tail is promoted by phosphorylation of LRP6. Phosphorylation of LRP6 occurs on several clusters of serines and threonines, with a central PPPSP motif as a hallmark. The serine in the PPPSP motif is modified by GSK3, leading to activation of signaling [29]. In addition, a member of the CK1 family, CK1 gamma, phosphorylates residues next to the PPPSP motif [30]. CK1 gamma, interestingly, has a membrane anchor in the form of a palmitoylation domain.

There are other proteins with known Wnt-binding domains that can serve as receptors for Wnt ligands. The single-pass tyrosine kinase Ror2, although structurally distinct from $\mathrm{Fz}$ receptors, is involved in other forms of Wnt signaling [31-35]. In the mouse, Ror2 and Ror1 knockout phenotypes resemble those of Wnt5a-/- null mice [36, 37]. In line with this common phenotype is the finding that Ror2 can mediate the Wnt5a signal that is responsible for inhibition of $\beta$-catenin-TCF signaling [31]. Thus, the CRD domain may enable other alternative signaling cascades to be initiated in response to Wnt ligand stimulation.

Another well-characterized Wnt-binding domain is the 
Wnt inhibitory factor (WIF) module, which is also found in the cell surface atypical receptor tyrosine kinase Ryk [38]. In Drosophila, the Ryk ortholog Derailed binds to Wnt5 to control commissural axon guidance in a potentially non-canonical fashion [39]. In mammalian systems, Ryk is required for Wnt3a-mediated canonical Wnt signaling [40]. Understanding the ways in which alternative Wnt receptors such as Ryk and Ror2 interact with known Wnt signaling components and what intracellular signaling cascades they initiate will lead to exciting advances in the field.

\section{Wnt signaling and embryonic stem cells}

Since the isolation of human ES cells, the use of ES cells as a regenerative tool has become closer to reality. But a problem to date is that most embryonic stem cells (mouse and human) are cultured in the presence of mouse embryonic fibroblasts (MEFs), serum and other animal products, which can introduce unwelcome pathogens into the ES cell culture. To be able to culture ES cells animal product-free, it is of critical importance to define all factors that maintain ES cells in an undifferentiated state. Although progress has been made toward culturing mouse and human ES cells serum- and feeder-free, only a few of the presumably many factors have been defined, such as leukemia inhibitor factor (LIF) and BMP4 for mouse and basic fibroblast growth factor for human ES cells [41]. Given its prominent role in adult stem cell systems, it is plausible that Wnt signaling plays a role in ES cell culture. Although recent reports indeed implicate Wnt signaling in keeping ES cells pluripotent, contradicting results have been published [42]. Consequently, further research is necessary to define the exact role of Wnt signaling in ES cell maintenance.

There are many lines of evidence, mostly coming from mouse ES cell research, that Wnt signaling components are involved in ES cell control. For example, Pereira et al. showed that TCF3, a Wnt controlled transcription factor, repressed nanog, one of the genes required for ES cell self-renewal [43]. Furthermore, Jeanisch and co-workers have shown that overexpression of Oct-4, another selfrenewing gene, causes dysplastic lesions of progenitor cells and increased $\beta$-catenin transcriptional activity [44]. Other evidence includes that activation of Wnt signaling, by genetically eliminating the function of the negative regulator APC, promotes the undifferentiated phenotype of mouse ES cells [42].

\section{Wnt signaling and neural stem cells}

During the development of the nervous system, primitive cells act as a source of various types of specialized cells that make up the functioning brain. Neurons, astrocytes and oligodendrocytes are derived a single type of precursor cell. Several studies have suggested that these precursor cells are able to self-renew, a hallmark of stem cells [45]. Neural stem cells are also implicated in adult neurogenesis, a process that is restricted to two regions of the central nervous system: the subventricular zone of the lateral ventricle and the subgranular zone of the hippocampal dentate gyrus. In the embryo, neurogenesis is likely to be less restricted anatomically; but, in both cases, signals provided by the microenvironment regulate the maintenance, proliferation and neuronal fate commitment of the local stem cell populations. While much effort has been devoted to understanding the development of the central nervous system in both the adult and embryonic settings, the identity of the signals regulating neurogenesis is largely unknown. Identifying these factors may increase opportunities to grow and expand neural stem cells in culture, a prerequisite for tissue engineering.

In the nervous system, phenotypes of mouse Wnt mutants, the Wnt1 knockout which results in loss of midbrain for example, suggest that Wnts are candidates for neural stem cells' self-renewal and are major players in blocking their differentiation. Wnt3a mutant mice exhibit underdevelopment of the hippocampus due to lack of proliferation [45], and recent work demonstrating enhanced neurogenesis in vivo via exogenous expression of Wnt3a via lentiviral vectors strengthens the model that the Wnt signaling pathway is a major regulator of adult neurogenesis in the hippocampus [46]. A $\beta$-catenin gain-of function study by Walsh et al. shows that continuous Wnt signaling results in expansion of the brain [47]. Together, these studies imply that using the Wnt pathways in regeneration of cells in the nervous system will be an exciting area of research.

\section{References}

1 Korinek V, Barker N, Moerer P, et al. Depletion of epithelial stem-cell compartments in the small intestine of mice lacking Tcf-4. Nat Genet 1998; 19:379-383.

2 Reya T, Duncan AW, Ailles L, et al. A role for Wnt signalling in self-renewal of haematopoietic stem cells. Nature 2003; 423:409414.

3 Willert K, Brown JD, Danenberg E, et al. Wnt proteins are lipidmodified and can act as stem cell growth factors. Nature 2003; 423:448-452.

4 Bienz M, Clevers H. Linking colorectal cancer to Wnt signaling. Cell 2000; 103:311-320.

5 Jamieson $\mathrm{CH}$, Ailles LE, Dylla SJ, et al. Granulocyte-macrophage progenitors as candidate leukemic stem cells in blast-crisis CML. N Engl J Med 2004; 351:657-667.

6 Logan CY, Nusse R. The Wnt signaling pathway in development and disease. Annu Rev Cell Dev Biol 2004; 20:781-810.

7 Nusse R, Varmus HE. Wnt genes. Cell 1992; 69:1073-1087.

8 Miller JR. The Wnts. Genome Biol 2002; 3:REVIEWS3001. 
9 Mason JO, Kitajewski J, Varmus HE. Mutational analysis of mouse Wnt-1 identifies two temperature-sensitive alleles and attributes of Wnt-1 protein essential for transformation of a mammary cell line. Mol Biol Cell 1992; 3:521-533.

10 Reichsman F, Smith L, Cumberledge S. Glycosaminoglycans can modulate extracellular localization of the wingless protein and promote signal transduction. J Cell Biol 1996; 135:819-827.

11 Tanaka K, Kitagawa Y, Kadowaki T. Drosophila segment polarity gene product porcupine stimulates the posttranslational N-glycosylation of wingless in the endoplasmic reticulum. J Biol Chem 2002; 277:12816-12823.

12 Takada R, Satomi Y, Kurata T, et al. Monounsaturated fatty acid modification of Wnt protein: its role in Wnt secretion. Dev Cell 2006; 11:791-801.

13 Ching W, Nusse R. A dedicated Wnt secretion factor. Cell 2006; 125:432-433.

14 Bänziger C, Slodini D, Schütt C, et al. Wntless, a conserved membrane protein dedicated to the secretion of Wnt proteins from signaling cells. Cell 2006; 125:509-522.

15 Bartscherer K, Pelte N, Ingelfinger D, Boutros M. Secretion of Wnt ligands requires Evi, a conserved transmembrane protein. Cell 2006; 125:523-533.

16 Panakova D, Sprong H, Marois E, Thiele C, Eaton S. Lipoprotein particles are required for Hedgehog and Wingless signalling. Nature 2005; 435:58-65.

17 Verges M, Luton F, Gruber C, et al. The mammalian retromer regulates transcytosis of the polymeric immunoglobulin receptor. Nat Cell Biol 2004; 6:763-769.

18 Coudreuse DY, Roel G, Betist MC, Destree O, Korswagen HC. Wnt gradient formation requires retromer function in Wnt-producing cells. Science 2006; 312:921-924..

19 Prasad BC, Clark SG. Wnt signaling establishes anteroposterior neuronal polarity and requires retromer in C. elegans. Development 2006; 133:1757-1766.

20 Bhanot P, Brink M, Samos CH, et al. A new member of the frizzled family from Drosophila functions as a Wingless receptor. Nature 1996; 382:225-230.

21 Hsieh JC, Rattner A, Smallwood PM, Nathans J. Biochemical characterization of Wnt-frizzled interactions using a soluble, biologically active vertebrate Wnt protein. Proc Natl Acad Sci USA 1999; 96:3546-3551.

$22 \mathrm{Wu} \mathrm{CH}$, Nusse R. Ligand receptor interactions in the Wnt signaling pathway in Drosophila. J Biol Chem 2002; 277:4176241769.

23 Chen W, ten Berge D, Brown J, et al. Dishevelled 2 recruits betaarrestin 2 to mediate Wnt5A-stimulated endocytosis of Frizzled 4. Science 2003; 301:1391-1394.

24 Wehrli M, Dougan ST, Caldwell K, et al. Arrow encodes an LDL-receptor-related protein essential for Wingless signalling. Nature 2000; 407:527-530.

$25 \mathrm{Li} \mathrm{Y,} \mathrm{Bu} \mathrm{G.} \mathrm{LRP5/6} \mathrm{in} \mathrm{Wnt} \mathrm{signaling} \mathrm{and} \mathrm{tumorigenesis.} \mathrm{Fut}$ Oncol 2005; 1:673-681.

26 He X, Semenov M, Tamai K, Zeng X. LDL receptor-related proteins 5 and 6 in $\mathrm{Wnt} /$ beta-catenin signaling: arrows point the way. Development 2004; 131:1663-1677.

27 Liu G, Bafico A, Harris VK, Aaronson SA. A novel mechanism for Wnt activation of canonical signaling through the LRP6 receptor. Mol Cell Biol 2003; 23:5825-5835.

28 Mao J, Wang J, Liu B, et al. Low-density lipoprotein receptor- related protein- 5 binds to Axin and regulates the canonical Wnt signaling pathway. Mol Cell 2001; 7:801-809.

29 Zeng X, Tamai K, Doble B, et al. A dual-kinase mechanism for Wnt co-receptor phosphorylation and activation. Nature 2005; 438:873-877.

30 Davidson G, Wu W, Shen J, et al. Casein kinase 1 gamma couples Wnt receptor activation to cytoplasmic signal transduction. Nature 2005; 438:867-872.

31 Mikels AJ, Nusse R. Purified Wnt5a protein activates or inhibits beta-catenin-TCF signaling depending on receptor context. PLoS Biol 2006; 4:e115.

32 Forrester WC. The Ror receptor tyrosine kinase family. Cell Mol Life Sci 2002; 59:83-96.

33 Matsuda T, Nomi M, Ikeya M, et al. Expression of the receptor tyrosine kinase genes, Ror1 and Ror2, during mouse development. Mech Dev 2001; 105:153-156.

34 Oishi I, Suzuki H, Onishi N, et al. The receptor tyrosine kinase Ror2 is involved in non-canonical Wnt5a/JNK signalling pathway. Genes Cells 2003; 8:645-654.

$35 \mathrm{Xu}$ YK, Nusse R. The Frizzled CRD domain is conserved in diverse proteins including several receptor tyrosine kinases. Curr Biol 1998; 8:R405-R406.

36 Yamaguchi TP, Bradley A, McMahon AP, Jones S. A Wnt5a pathway underlies outgrowth of multiple structures in the vertebrate embryo. Development 1999; 126:1211-1223.

37 Yoda A, Oishi I, Minami Y. Expression and function of the Rorfamily receptor tyrosine kinases during development: lessons from genetic analyses of nematodes, mice, and humans. J Recept Signal Transduct Res 2003; 23:1-15.

38 Kroiher M, Miller MA, Steele RE. Deceiving appearances: signaling by "dead" and "fractured" receptor protein-tyrosine kinases. Bioessays 2001; 23:69-76.

39 Yoshikawa S, McKinnon RD, Kokel M, Thomas JB. Wnt-mediated axon guidance via the Drosophila Derailed receptor. Nature 2003; 422:583-588.

$40 \mathrm{Lu} \mathrm{W}$, Yamamoto V, Ortega B, Baltimore D. Mammalian Ryk is a Wnt coreceptor required for stimulation of neurite outgrowth. Cell 2004; 119:97-108.

41 Ludwig TE, Levenstein ME, Jones JM, et al. Derivation of human embryonic stem cells in defined conditions. Nat Biotechnol 2006; 24:185-187.

42 Kielman MF, Rindapää M, Gaspar C, et al. Apc modulates embryonic stem-cell differentiation by controlling the dosage of beta-catenin signaling. Nat Genet 2002; 32:594-605.

43 Pereira L, Yi F, Merrill BJ. Repression of nanog gene transcription by tcf3 limits embryonic stem cell self-renewal. Mol Cell Biol 2006; 26:7479-7491.

44 Hochedlinger K, Yamada Y, Beard C, Jaenisch R. Ectopic expression of Oct-4 blocks progenitor-cell differentiation and causes dysplasia in epithelial tissues. Cell 2005; 121:465-477.

45 Lee SM, Tole S, Grove E, McMahon AP. A local Wnt-3a signal is required for development of the mammalian hippocampus. Development 2000; 127:457-467.

46 Lie DC, Colamarino SA, Song HJ, et al. Wnt signalling regulates adult hippocampal neurogenesis. Nature 2005; 437:13701375.

47 Chenn A, Walsh CA. Regulation of cerebral cortical size by control of cell cycle exit in neural precursors. Science 2002; 297:365-369. 